\title{
Shaping the Ambience of Homes with Domestic Hypermedia
}

\author{
Marianne Graves Petersen and Kaj Grønbæk \\ Center for Interactive Spaces \\ Department of Computer Science \\ University of Aarhus \\ Åbogade 34, DK-8200 Århus N \\ \{mgraves,kgronbak\}@interactivespaces.net
}

\begin{abstract}
We currently witness a massive digitization of domestic materials e.g. photos, music, calendars, recipes, notes, messages. This digitization provides new conditions for how we interact with materials as well as how users shape the ambience of their homes. Observing the qualities of physical materials in the home, the process of digitization risks loosing the qualities of the spatial distribution, the aesthetics, and common reference points offered by physical materials and places in the home. We present a concept of domestic hypermedia (DoHM), which exploits potentials of digital materials and at the same time allow people to interact with digital materials in engaging ways providing rich experiences when organizing and using digital materials in homes. We present an infrastructure and design concepts that offer: ambient access to digital materials, common reference points, and collective experiences.
\end{abstract}

\section{Keywords}

Ubiquitous hypermedia, domestic technology, augmented reality, context awareness.

\section{Introduction}

We currently see an increased digitization of domestic material. Photos, movies, calendars, recipes, notes, banking, messages from school etc. increasingly become digitized and thus no longer have an inherent physical form [24]. Historically, the workplace has undergone a similar transition; however, the home is quite different from the workplace in many respects [3]. Activities in the home are less task-oriented [17]; the rationalities of work in terms of production, efficiency and organization of labor do not necessarily transfer to the home [3]. Moreover inhabitants continuously re-configure and appropriate their homes both to express their identity to the outside world [20], but equally to capture their own history and biography [26] below. Thus homes have their own aesthetics where the visible, physical "information material" often play a role in expressing identity e.g. in terms of the books being read or the music listened to by the inhabitants. These qualities of the domestic environment are important to understand when designing hypermedia for the home. 
Thus in order to design future domestic hypermedia systems, we argue that it is necessary to study the characteristics of the existing use of both physical and digital materials in homes today. While we are not the only one to take this point of stance, our focus is different from other studies. E.g. some groups have focused on understanding patterns of domestic routines ([3], [2]) where we have focused more broadly on eliciting the different roles domestic information materials may have, not restricted to patterns of routine. Others have investigated what constitutes the home experience [5] more generally, but this study did not reveal how specific materials formed part of constituting the home experience. Our studies are inspired by the studies by [3][2], who investigate how the physical space of the home is used to coordinate the handling of paper mail, but our focus is on domestic materials beyond paper mail. The mission of this paper is to learn how to take the best from both the digital and physical sides and combine into concepts for future domestic hypermedia systems.

\section{Empirical Studies}

In the following, we describe the approach taken in studying domestic hypermedia and we provide examples of the information materials, which exist in private homes. We have undertaken qualitative empirical studies in six private homes inhabited by different types of families, ranging from singles, over families with children living at home, to communes. We visited each household once and stayed for approximately one and a half to two hours each place. In each home, we asked the inhabitants to take us on tours around the house [22]. We focused on how people organize domestic information materials in general, i.e. media, letters, memo lists, newspapers etc., and we interviewed people about the rationale, and history of the physical placements of both physical and digital materials. We asked about ownership and possible conflicts around placement of materials [19]. We captured data from the homes through video recordings, and pictures. The six visits form part of a continued investigation into domestic technology use of our own [21], [13] as well as others ([3], [19]).

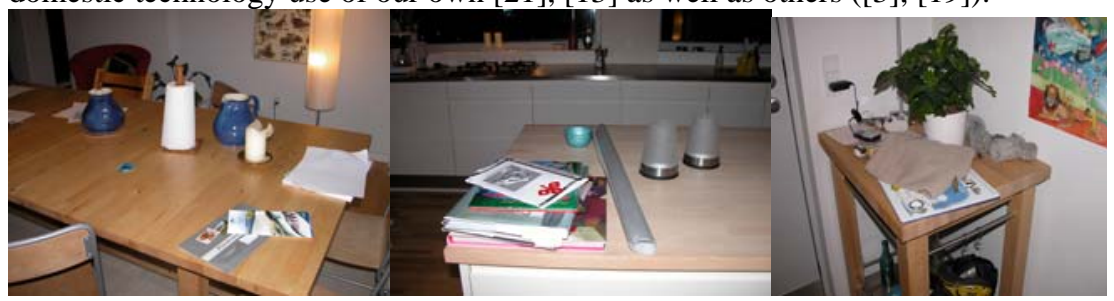

Figure 1: Left: Dining table with brochure from a theme park recently visited. Middle: Mixed pile of children's' book, adults' book, and unused wrapping paper awaiting further distribution in the home. Right: Table in Entrance hall with a pile of things to remember when leaving the house.

\section{Domestic surfaces}

Crabtree et al. [3] were among the first to spell out in details how the specific surfaces in the home designate certain meaning and serve to coordinate domestic life. In their study they focused on paper mail (ibid), and described how if for instance a letter is 
found by the porch, it suggests that no other person has dealt with it yet. Confirming the studies of Crabtree et al., but focusing on domestic information materials more broadly, our studies also suggest that the spatial layout of the home is indeed used to coordinate and structure domestic information materials.

Figure 1 illustrates how different placement of physical materials in the house have different meanings and how the spatial layout serves structure domestic information material, e.g. the material on the table by the front door is placed there to remind the user to bring it when leaving the home. To illustrate the range of different surfaces and their characteristics table 1 provides a set of examples from the homes visited.

Table 1: Examples of different surfaces and their characteristics

\begin{tabular}{|c|c|}
\hline Surface & Surface characteristics \\
\hline $\begin{array}{l}\text { Notice } \\
\text { boards }\end{array}$ & $\begin{array}{l}\text { Information which is ‘one click away' } \\
\text { Contact information, Notifications, Memorable material }\end{array}$ \\
\hline Table & $\begin{array}{l}\text { Traces of earlier activities } \\
\text { Piles awaiting further distribution in the home } \\
\text { Mixed media activity spaces }\end{array}$ \\
\hline Entrance hall & Things to remember to bring along when leaving the home \\
\hline Shelves & $\begin{array}{l}\text { Media collections. Not always sorted optimally for searching, but rather in terms of } \\
\text { aesthetics and of expressing identity. }\end{array}$ \\
\hline Frames & Staging of personal memory \\
\hline
\end{tabular}

We also see how other interests than supporting browsing and searching come into play, when placing materials in homes. E.g. as illustrated in Figure 2, only selected cookbooks are placed so they are immediately visible to visitors whereas the rest of the collection is kept in a place where visitors not normally come.

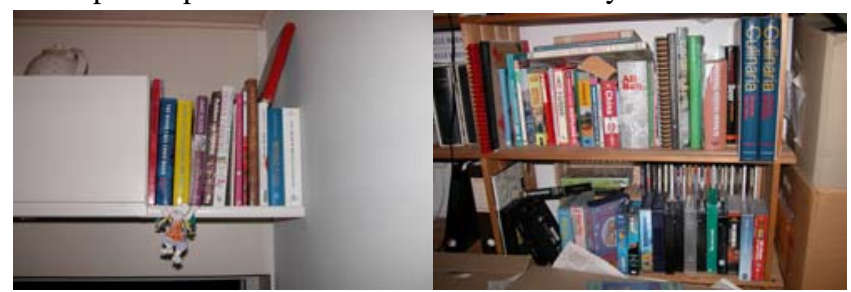

Figure 2: Left: Shelves in kitchen where selected cookbooks are on display. Right Shelves in home office, holding the other part of the cookbook collection.

This is an example of what Palasmaa has described as "Home is a staging of personal memory. Personal space expresses the personality to the outside world, but equally important, it strengthens the dweller's self-image and concretizes his world order" ([20], p. 6). As homes become meaningful in this way, it is important to ensure that this is also supported in future domestic environments where domestic materials are digitized. The highly spatial distribution of physical materials onto a multitude of surfaces in homes stands in marked contrast to what happens, as domestic materials become digitized and stored on a personal computer in the home. The PC essentially provides centralized and individualized access to digital materials. It lacks the spatial 
distribution, the persistence and visibility of physical materials on display in the home, as we see numerous examples of in our empirical material. Thus we need to learn from the qualities of physical material, which enable spatial information structuring in the home when designing domestic hypermedia systems.

\section{Lazy structuring}

A further lesson from studying physical materials is how it allows unfinished and "lazy" structures. This can be seen from the widespread use of piles as a structuring mechanism. As illustrated in figure 3, all the homes we visited had piles. For instance piles of bank papers and official papers waiting to be filed in binders.

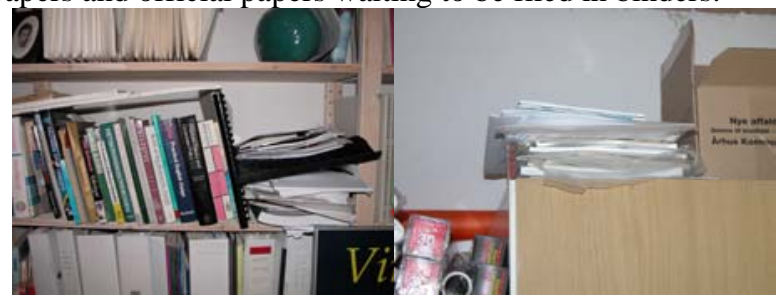

Figure 3: Piles as structuring mechanism in homes

Piles are not as unstructured as it may first seem thanks to the meaning of different surfaces of the home, as discussed above. Some are piles of heterogeneous material, implicitly ordered by date, as sedimentations of materials. Not an effective structuring mechanism for a fast search, but a mechanism, which with the least possible effort still puts some structure on the material, largely due to the specific location of the pile. The pile in the entrance hall in Figure 1 is not sediment. It is carefully selected stuff which must be brought when leaving the home etc. Thus the specific context of the pile provides an additional layer of meaning to the structure. This dimension is lost, if most domestic material can only be distributed on one, digital desktop.

\section{The structuring experience}

While one way to be pragmatic with respect to everyday life is to support lazy structuring mechanisms, an alternative and complementary approach is to design for playful and engaging structuring experiences thus aspiring people to take time to impose structure on their materials. Given the nature of the home, it would be interesting to investigate how collective and more playful structuring experiences can be supported. A topic we address in our design concepts, presented in the following.

\section{Domestic Hypermedia - Folding Hyperspaces Into the Home}

The empirical studies point to a lot of different ways of organizing physical material in the home. However, collaborative spatial organization and persistent visual awareness of the material in specific places seem to be most at risk in the process of digitization of domestic materials. In this section, we propose a domestic hypermedia infrastructure that supports these approaches to structuring digital material. We propose a hypermedia concept, where digital hyperspaces are folded into the physical space. The notion of folding is inspired by the work on folding within architecture. In 
this tradition, folding is a means to create more dynamic and open buildings by folding rooms of one type in between rooms of another type. As described by Lynn [14] below, folding employs neither agitation nor evisceration but a supple layering. In such a way, that the characteristics of the individual layers are maintained. "A folded mixture is neither homogeneous, like whipped cream, nor fragmented like chopped nuts, but smooth and heterogeneous” (ibid p.9).

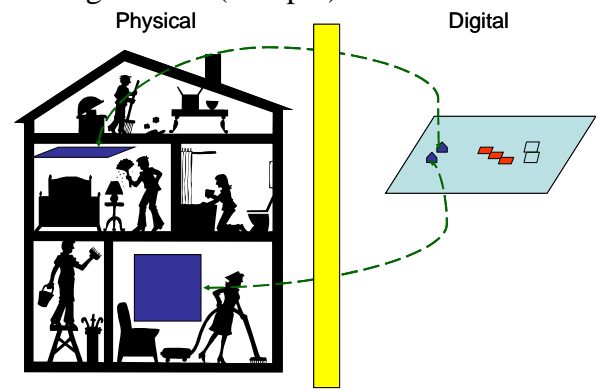

Figure 4: Folding physical and digital spatiality

We propose domestic hypermedia as the folding of digital spaces with physical spaces in a way where the characteristics of both spaces are preserved, yet they are connected through layering. Thus we envision a multitude of digitally enabled surfaces in the future home. Each surface resides in a particular physical context, in the digital environment. The different "surfaces" may with current technology be TVs, projections, computer displays, mobile phones, as well as HiFi equipment with only an auditory appearance. In the future, we envision surfaces in more persistently visible materials like Gyricon [9] and eInk [6] paper, which is material allowing persistent display, also when the power is turned off. In addition we may see a variety of ambient displays and controls [29] like wallpaper, bottles, RFID readers etc.

\section{Domestic Hypermedia Infrastructure}

The DoHM domestic hypermedia infrastructure (see Figure 5) is built on top of the open context-aware hypermedia framework HyCon [1]. HyCon is a context-aware hypermedia framework with a generic sensor layer that enables hypermedia clients to sense the context of users and devices registered in the framework. Moreover, HyCon supports XLINK structures, RDF, annotations, and WebDAV servers for collaborative handling of content and structures. HyCon among other things supports hypermedia composites utilizing XLINK.

DoHM is in particular designed to support folding of hyperspaces into the physical space. The empirical studies show that spatial organization and piling of material are common structuring mechanism in homes as well as lazy or incomplete structuring is observed in many places. Thus DoHM supports spatial hypermedia structuring integrating the connected physical surfaces as first class composite structures. The DoHM infrastructure also supports carrying digital material around both inside and outside the home by means of having a physical token (like a cell phone or a smartcard) representing a person's digital portfolio on the home server and other integrated servers. 


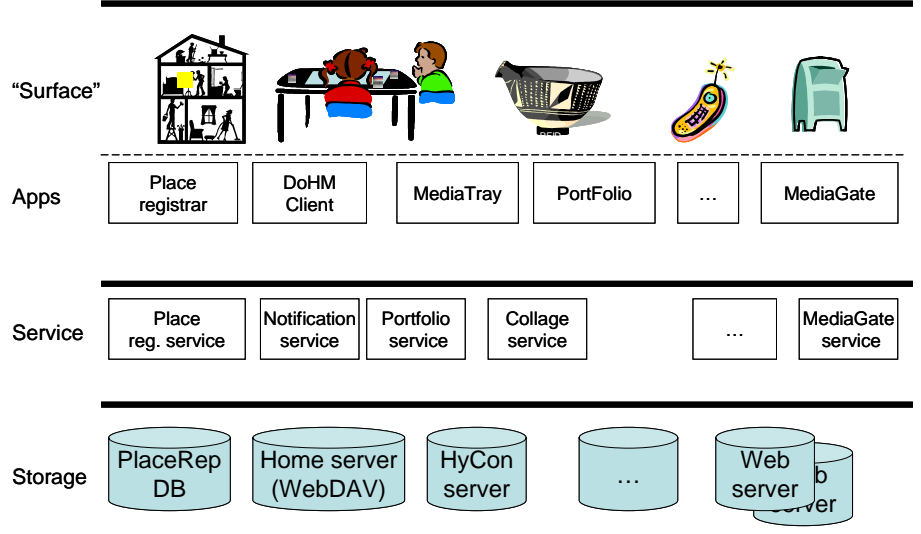

Figure 5: DoHM - Domestic Hypermedia infrastructure

The DoHM infrastructure is thus capable of handling the common structures that are used in homes, e.g. composites representing play lists, photo albums, and cookbooks. Meta-data associated with physical objects or digital material like photos are represented in RDF. A central user interface for DoHM is the client, which provides a 2D spatial hypermedia interface including the registered surfaces as objects, as illustrated in figure 5 .

\section{Modeling home surfaces}

The various surfaces in the private home needs to be addressable from the point of view of the hypermedia system, such that particular material can be presented in different or even multiple physical locations in the home. As discussed in section 3.2 surfaces in the private home serve many different purposes and exist in different contexts. E.g. things to remember when leaving the home are placed persistently and visibly in the hallway. Peripheral awareness of earlier activities is created through the traces of material left on dining table.

This is implemented in DoHM through adding two central concepts to the HyCon framework [1] in order to handle places and presentation. PlaceReps represent the place or context of a particular domestic hypermedia surface in the home; and PresStyles represent the style specification for a given object or surface.

\section{PlaceReps and PresStyles}

PlaceReps may represent both a specific place like "the refrigerator door" or an abstract place like "entrance" which may cover several physical locations in the home including, both hall, basement and terrace. Thus in this way, information that must be recalled when leaving the home can be displayed in all the physical entrances.

In light of the nature of the different materials and surfaces (table 1), some material on some surfaces should be able to call for attention in some contexts, e.g. an appointment with the dentist, whereas others need to be discrete and aging, e.g. pictures from a recent weekend trip with friends. Thus we wish to support the users in specifying different presentation styles for material and surfaces in the home. We introduce the PresStyle class to represent the specific presentation style for a material object or 
a surface object. It holds an Id, a name, presentation specification, a priority and an optional script. Both surfaces and material objects may be associated with a PresStyle; this introduces the need for a combination strategy similar to the issues of Dexter Pspecs [7] and the cascade of Cascading style sheets [4].

We wish to give users maximum freedom in presenting digital material, to support the continuous appropriation of the home [26]. For instance, a teenage kid may prefer to have a certain skin PresStyle associated with the surfaces reminding her of a favorite movie on every surface in the room. On the other hand, parents may want to associate an intrusive red colored notification PresStyle with a reminder note that they place on every relevant surface in the home. In this case there should be a combination strategy that allows both the teenager to maintain her surface skin and to be notified about the important note from the parents. This is solved by a calculation of the cascade based on the cascade priority attributes of the involved PresStyles.

Based on the empirical studies we have identified a number of relevant PresStyle specifications to be supported. Examples of PresStyles being supported are: Persistent, Aging, Collage, Emphasize, Surprise, Animated, Conditional Appearance, and Context Sensing. Most of these styles apply to both object and the surface per se, but for instance Emphasize is only applicable to single objects since the semantics is to highlight an individual object. The first four PresStyles directly follow from the way people organize themselves with physical materials in homes. The latter explores the new possibilities opened up by having dynamic, context aware digital surfaces in the homes. The PressStyles are subject to further experimentation and evaluation in an iterative design process.

\section{Appliances Utilizing The DoHM Infrastructure}

In this section we describe some design concepts that provide new structuring experiences in homes as well as new means of shaping the ambience of homes. All are built upon the DoHM system

\section{MediaGates for piles of unsorted incoming material}

DoHM provides support for uploading of digital material and scanning of physical material to the home server. The gate hardware will typically be scanners (for paper), FlashRam or USB ports (for pictures and documents), FireWire ports (for video). But email, SMS/MMS messages may also be transferred to gate space. The material being uploaded will be dropped onto a "gate" folder and canvas acting as temporary space for unsorted incoming material. This gate canvas can be associated with places in the home, where the family wish to create awareness of recent un-processed material. One such place may be the dining table which in homes is often used as a gate area for physical material in transition into other places in the home, e.g. see Figure 1. In this way we may support the lazy structuring witnessed in homes, as seen in Figure 3.

\section{MediaTable: Augmented collaborative dining table}

In many families, the dining table is a central place for coordinating activities, exchanging messages, and for organizing incoming materials [3]. In the studies we have seen how families leave physical material on the dining table or the common room 
table creating a shared awareness in the home, before it is put away on a shelf or pinned to a notice board. When physical material becomes digitized it is often uploaded to or received on a computer somewhere in the home by one family member without reaching the attention of the rest of the family.

In a future home with many digital materials, we wish to be able to support collective coordination and organization of materials similar to the physical case. We are thus designing the DoHM client to support e.g. a dining table to be augmented with top projection and direct tangible interaction. The DoHM client present itself to the user as a spatial hypermedia interface, implemented in SVG thus allowing PlaceReps, interaction elements and material objects to be turned around to be visible from arbitrary positions around the table, see Figure 6 . A common room table may become a digital surface providing touch based interaction. The default view is the scratch area with incoming material and visual icons for the registered PlaceRep objects.

In this way, family members become aware of new materials like incoming messages, new photos, new MP3s etc. Family members passing by can for instance drag a piece of material to a specific PlaceRep icon to make the material visible one or more surfaces in the home shown with the default PresStyle for these surfaces.
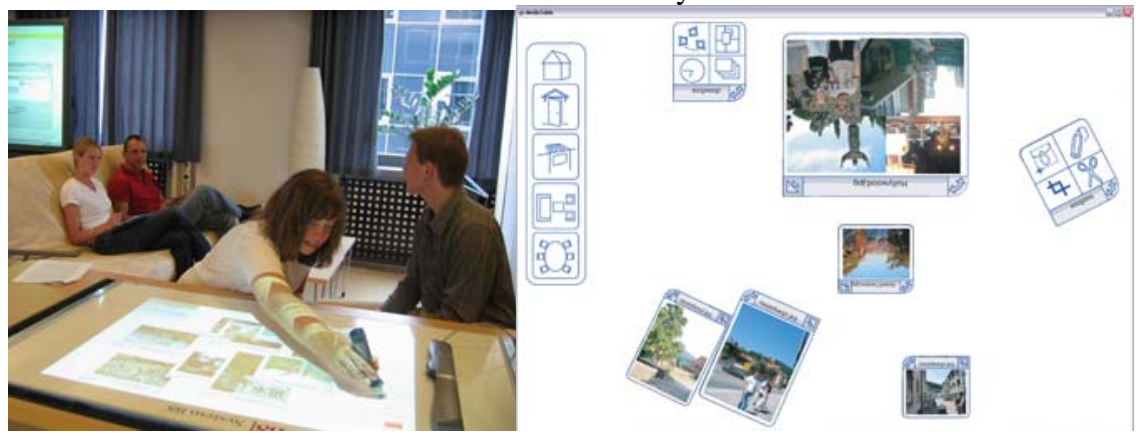

Figure 6: Left: A scenario with two people using the MediaTable pushing pictures to the TV "surface" being watched from the sofa. Right: The DoHM client supporting a collaborative picture sorting scenario. Icons on the left are PlaceRep icons. Floating toolbars support object interaction.

In a situation where the family gathers in the living room the members may sit down or stand around the table and sort the piles of recent pictures into folders on the home server and drag some of them to specific PlaceReps to have them rendered on specific surfaces. They may also associate specific digital material with physical objects by means of RFID tags as described in [7]. The objects may be printouts of pictures or souvenirs like a concert ticket or a kids' doll, which then becomes a physical link anchor for a picture or a collection of pictures. Thus the table also aims to support collective organization of material thus supporting new types of structuring experiences as suggested in section 3.4.

\section{MediaWall: Calm awareness support}

In order to exploit the potential of surfaces in the home, we have developed the concept of the MediaWall. The MediaWall offers the quality of persistently visible message in specific positions, which is preferred in many cases. Maintaining aware- 
ness about important material or critical events is supported in the DoHM system through discrete or calm [28] awareness based on discrete visual appearance. We are inspired by the notions of Informative arts such as the work by PLAY [25] as well as InfoCanvas [16]. The idea is to provide awareness about important material through an artistic collage of the material on one or more surfaces. To support this we take advantage of the open hypermedia techniques for anchoring links in arbitrary Web pages. Here we let family members make selections on arbitrary Web pages including the home server. They can then submit the corresponding anchors to a collage service together with a PlaceRep and a PresStyle. The surface identified by the PlaceRep will then display the selected text and graphics corresponding to the anchor according to some schema with an expression designed to fit the actual room and the inhabitant's preferred level of calmness.

The collage service is meant to maintain an ongoing awareness of the updated view of information identified by the submitted anchors. An active crawler is needed to regularly collect information from servers, filtering the parts of the pages needed, making an artistic transformation before the representation is dispatched to the surfaces pointed out by the PlaceReps that were associated with the anchor when submitted to collage service.

The MediaGate, the MediaTable, and the MediaWall together present a vision of a domestic hypermedia system, which takes into account the specific challenges of the household. The MediaGate addresses the challenge of lazy structuring, the MediaTable offers new structuring experiences, and the MediaWall takes advantage of the meaning and richness of the surfaces of the home. We currently have a first implementation of the key aspects of the three appliances. These will be subject to experiments and further evaluations in real life homes before a next version is developed.

\section{Related Work}

While others have conducted research into future interactive home environments, our work differs in various ways from previous. Compared to the studies by Crabtree [2], we have covered a broader range of material and media than just the physical mail. Compared to related design concepts, we focus on supporting the handling of materials among people who actually live in the same home, rather than supporting awareness between people living in different physical locations, which has been investigated by other projects [18][12]. Moreover, we focus on handling of digital materials broader than photos [27] and we challenge the position that experiences of handling digital photos in homes can be limited to searching, wandering and recommending [27]. In contrast, we suggest that digital photos may be important material in shaping the ambience of homes, provided that interaction mechanisms are supported that respect the qualities of the homes. Our first suggestion is in the form of collective and playful experiences around the MediaTable, which supports collective handling of materials, which is in opposition to the prevalent, more individualized concepts [27].

Compared to the Jigsaw domestic component system [11], we have taken a material centered approach. We have focused on the organization of domestic material and on how we can provide a seamless folding between the physical and digital material 
spaces. In achieving that we introduce a novel combination of open hypermedia, spatial hypermedia, physical hypermedia, and context-awareness in the DoHM infrastructure. Where Humble et al. [11] focus on supporting transformations between digital and physical material, we focus on linking and integrating the physical and the digital. We do this in terms of the home environment per se by developing support for the DoHM system which directly addresses surfaces in the home and let users address arbitrary surfaces from every DoHM client running in the home.

LiMe[23] is a Philips project among other things developing a CafeTable and Public Screen concept with access to digital material and the ability to relate it to RFID tags. Compared to LiMe, DoHM focuses on the collaborative interaction with home materials, and the distributed management of materials on surfaces in the home.

Spatial hypermedia systems ([8], [15]) have been a source of inspiration, and we have extended spatial hypermedia with abstract representations (PlaceReps) of physical surfaces and places. A PlaceRep is associated with the composite which holds the collection of material to be presented according to the default behavior (specified by PresStyles). Thus selected composites in the DoHM system are continually connected to physical place or surface.

In the paper [8], we discuss outdoor geo-spatial hypermedia, and we make a distinction between metaphorical and literal spatiality. The DoHM system combines support for these distinctions. The PlaceReps are literal in the sense that they represent an actual physical place or surface in the home. But it is a deliberate choice not to have an exact 2D or 3D model of the house to deal with, since that is far too complex for an action putting a picture on a specific notice board. Thus we view PlaceReps slightly more metaphorical in the sense that we can deal with e.g. "entrance” and have that cover a list of physical locations in the home. This supports inhabitants in developing a set of PlaceReps that makes sense to them. In the architectural folding terminology, we support the inhabitants in tailoring their own folding of the digital environment into the set of installed digital surfaces in the physical environment.

\section{Conclusions}

In this paper we have presented studies of the use of primarily physical material in private homes. We have illustrated how taking the context of the home seriously implies certain challenges for domestic hypermedia. Designing for the pragmatics of real domestic life, rather than for some kind of idealized visions of human activities and domestic environments implies for instance supporting lazy structuring of domestic materials. It implies using the rich set of surfaces of the home as a resource in design, and it implies challenging the setting of the individualized personal computer and explores how collective and playful structuring mechanisms can be developed. Based on the challenges revealed in these studies we have described the design of a new Domestic Hypermedia infrastructure called DoHM, which supports the folding of spatial and navigational hypermedia into the physical environment of a home. We have presented a couple of novel home appliances that take advantage of the DoHM infrastructure. We have established the DoHM infrastructure and are initiating experiments with users introducing the appliances being designed. We have developed 
prototypes of the appliances and the DoHM infrastructure, and they will be evaluated both in our lab and in specific home settings within the coming months.

\section{Acknowledgements}

We thank our colleagues in Center for Interactive Spaces (www.interactivespaces.net) for their contributions to the work presented here, in particular we thank Bent G. Christensen and Martin Brynskov for their contribution to the development. Finally we thank the families who spent time with us and kindly let us into their homes.

\section{References}

[1] Bouvin, N.O., Christensen, B.G., Grønbæk, K., Hansen, F.A. HyCon: A Framework for Context-aware Mobile Hypermedia. 2003. To appear in NRHM journal volume 9.

[2] Crabtree, A., Hemmings, T. and Rodden, T. (2002) "Coordinate displays in the home”, CSCW Workshop on Public, Community and Situated Displays, Proceedings of the 2002 ACM Conference on Computer Supported Cooperative Work, New Orleans: ACM Press.

[3] Crabtree, A., Hemmings, T., and Rodden, T. (2002) Patternbased Support for Interactive Design in Domestic Settings. In Proc. of DIS2002, ACM Press, pp. 265-275.

[4] CSS. http://www.w3c.org/Style/CSS/

[5] Eggen, B., Rozendaal, M. and Schimmel, O. (2003). Home Radio - Extending the Home Experience beyond the Boundaries of the Physical House. In Proceedings of the Home Oriented Informatics and Telematics,University of California, Irvine, April 6-8, 2003.

[6] E-Ink. http://www.e-ink.com

[7] Grønbæk, K. \& Trigg, R.H. From Web to Workplace: Designing Open Hypermedia Systems. MIT Press, Boston Massechussets. July 1999, 424 pp (ISBN 0-262-07191-6).

[8] Grønbæk, K., Vestergaard, P.P., \& Ørbæk P. Towards Geo-Spatial Hypermedia: Concepts and Prototype Implementation. In proceedings of the $13^{\text {th }}$ ACM Hypertext conference, June 11th - 15th, 2002, University of Maryland, USA, ACM, New York, 2002.

[9] Gyricon SmartPaper. http://www.gyricon.com

[10] Holmquist, L. E., and Skog, T. (2003) Informative Art: Information Visualization in Everyday Environments. In Proc. of the first intl. conference in computer graphics and interaction techniques in Australia and Southeast Asia.

[11] Humble, J., Hemmings, T., Crabtree, A., Koleva, B. and Rodden, T. (2003) “"Playing with your bits': user-composition of ubiquitous domestic environments", Proceedings of the 5th international Conference on Ubiquitous Computing, pp. 256-263, Seattle: Springer.

[12] Hutchinson, H., Mackay, W., Westerlund, B., Bederson, B. B., Druin, A., Plaisant, C., Beaudouin-Lafon, M., Conversy, S., Evans, H., Hansen, H., Roussel, N., Eiderbäck, B. (2003) Domesticated Design: Technology Probes: Inspiring technology design with and for families. In proceedings of CHI 2003. ACM Press, pp. 17-24.

[13] Kjær, A., Madsen, K. H. \& Petersen, M. G. (2000) Methodological Challenges. In The Study Of Technology Use At Home. In Home Informatics and Telematics: Information, Technology and Society, Sloane, A. and van Rijn, F. (Eds.) Boston: Klüwer Academic Publ., pp.45-60 
[14] Lynn, G. (1994) Architectural curvilinearity : The Folded, the Pliant and the Supple. In Folding in Architecture. Architectural Design Magazine. Academy Grp Ltd., pp. 8-15.

[15] Marshall, C., \& Shipman, F. Spatial hypertext and the practice of information triage, In Proc. Tenth ACM Conference on Hypertext (Hypertext '97). (Southampton, UK, Apr, 1997), pp. 124-133.

[16] Millar, T., Stasko, J. (2001) The InfoCanvas: Information Conveyance through Personalized, Expressive Art. In Proceedings of CHI 2001, ACM Press, pp. 305-306.

[17] Monk, A. (2000) User-Centred Design. The Home use challenge. In Sloane, A. \& van Rijn, F. Home Informatics and Telematics. Information, Technology and Society. Kluwer Academic Publishers, pp. 181-190.

[18] Mynatt, E. D., Rowan, J., Craighill, S., and Jacobs, S. (2001) Digital Family Portraits: Supporting peace of mind of extended familiy members. In Proceedings of CHI2001, ACM Press, pp. 333-340.

[19] O’Brien, J., Rodden, T., Rouncefield, M., and Hughes, J. (1999) At Home with the Technology: An Ethnographic Study of a Set-Top-Box Trial. In ACM Transactions on Computer-Human Interaction, Vol. 6, No. 3, pp. 282-308.

[20] Pallasmaa, J. (1994) Identity, Intimacy and Domicile. Notes on the phenomenology of home. In Finnish Architectural Review 1 / 1994.

[21] Petersen, M. G., Madsen, K. H. and Kjær, A. (2002) Usability of Everyday Technology Emerging and Fading opportunities. In ACM Transactions on Computer-Human Interaction, Vol. 9, No. 2, June 2002, pp. 74-105.

[22] Petersen, M. G. and Baillie, L. (2001) Methodologies for Designing Future Household Technologies. In Proc. of the OIKOS2001 Workshop, Aarhus University Press, pp. 47-49

[23] Philips, LiMe project, http://www.design.philips.com/smartconnections/lime/

[24] Premkumar, G. P. (2003) Alternate distribution strategies for digital music. In Communications of the ACM, September, Volume 46, Issue 9.

[25] Redström J, Skog T \& Hallnäs L. Informative Art: Using Amplified Artworks as Information Displays. In Proceedings of DARE 2000, Designing Augmented Reality Environments, ACM, 2000, Elsinore, Denmark

[26] Silverstone, R., Hisch, E. \& Morley, D. (1992) Information and communication technologies and the moral economy of the household. In Consuming Technologies. Media and Information in Domestic Spaces. Routledge. London and New York. pp. 15-31.

[27] Teixeira, D., Verhaegh, W., and Ferreira, M. (2003) And Integrated Framework for Supporting Photo Retrieval Activities in Home Environments. In Lecture Notes in Computer Science. Issue 2875. Ambient Intelligence. Springer-verlag, pp. 288-303.

[28] Weiser, M. \& Brown, J.S. (July 1996) "Designing Calm Technology", PowerGrid Journal, v 1.01 .

[29] Wisneski, C., Ishii, H., Bahley, A., Gorbet, M., Braver, S., Ullmer, B., \& Yarin, P.: Ambient Displays: Turning Architectural Space into an Interface between People and Digital Information: Cooperative Buildings. Springer Verlag, February 25-26, 1998. 